\title{
01.5
}

\section{Ламинарный хаос в генераторе с запаздывающей обратной связью}

\author{
() Д.Д. Кульминский ${ }^{1,2}$, В.И. Пономаренко ${ }^{1,2}$, М.Д. Прохоров ${ }^{1}$ \\ ${ }^{1}$ Саратовский фолиал Института радиотехники и электроники им. В.А. Котельникова РАН, Саратов, Россия \\ ${ }^{2}$ Саратовский национальный исследовательский государственный университет им. Н.Г. Чернышевского, Саратов, Россия \\ E-mail: kulminskydd@gmail.com
}

Поступило в Редакцию 23 января 2020г.

В окончательной редакции 23 января 2020 г.

Принято к публикации 7 фревраля 2020г.

\begin{abstract}
Впервые экспериментально исследовано явление ламинарного хаоса в радиотехническом генераторе с запаздывающей обратной связью, время задержки которого модулируется внешним гармоническим сигналом. Построены области существования различных режимов ламинарного хаоса на плоскости параметров внешнего воздействия. Проведена реконструкция нелинейной функции генератора в режиме ламинарного xaoca.
\end{abstract}

Ключевые слова: генератор с запаздыванием, переменное время задержки, радиофизический эксперимент, ламинарный хаос.

DOI: 10.21883/PJTF.2020.09.49366.18218

Системы с запаздыванием чрезвычайно широко распространены в природе и технике. Для их описания обычно используются модели в виде дифференциальных уравнений с запаздывающим аргументом [1,2]. Такие модели включают в себя время задержки, которое позволяет учесть конечную скорость распространения сигнала в пространственно-развитых системах [3,4]. Наличие запаздывания, как правило, усложняет динамику системы и приводит к большему многообразию колебательных режимов. Так, системы с запаздыванием способны демонстрировать мультистабильность $[5,6]$, перемежаемость [7], состояния „химера“ $[8,9]$, фазовые переходы [10] и другие нелинейные явления [11], а использование систем с запаздыванием в системах связи позволяет повысить конфиденциальность передаваемой информации [12]. Большинство исследований систем с запаздыванием было проведено для случая постоянного времени задержки. В случае переменного времени задержки динамика системы становится еще более сложной $[13,14]$. Недавно в системах с изменяющимся временем задержки был обнаружен новый тип хаотического поведения, названный ламинарным хаосом $[15,16]$, при котором ламинарные фазы с практически постоянным значением динамической переменной периодически прерываются беспорядочными всплесками (берстами), переводящими систему из одной ламинарной фазы в другую, имеющую другое постоянное значение динамической переменной.

В настоящей работе явление ламинарного хаоса впервые исследовано в радиофизическом эксперименте на примере генератора с запаздывающей обратной связью. Впервые найдены области существования различных режимов ламинарного хаоса на экспериментальной плоскости параметров внешнего гармонического воздействия, модулирующего время запаздывания генератора.
Радиотехнические генераторы являются удобными объектами для экспериментального изучения различных феноменов нелинейной динамики в автоколебательных системах $[17,18]$. Такие генераторы могут быть относительно легко экспериментально реализованы, их динамика достаточно хорошо описывается известными модельными уравнениями, а их параметры можно легко менять в широких пределах. Для наблюдения ламинарного хаоса в эксперименте нами построен радиотехнический генератор с запаздывающей обратной связью, в котором реализована возможность модуляции времени задержки внешним гармоническим сигналом. Блок-схема экспериментальной установки показана на рис. 1.

Генератор представляет собой кольцевую систему, состоящую из линии задержки с переменным временем запаздывания, нелинейного элемента (NE) и низкочастотного $R C$-фильтра первого порядка. Линия задержки и нелинейный элемент генератора были реализованы в цифровом виде с помощью системы National Instruments PCI eXtensions for Instrumentation (NI PXI), а фильтр являлся аналоговым элементом. Аналоговые и цифровые элементы схемы сопрягались с помощью аналогоцифрового преобразователя ADC-1 и цифро-аналогового преобразователя DAC.

Время запаздывания линии задержки генератора изменялось от $\tau_{0}-\tau_{m}$ до $\tau_{0}+\tau_{m}$, где $\tau_{0}-$ среднее значение времени запаздывания, а $\tau_{m}$ характеризует глубину модуляции времени запаздывания. Для переключения времени запаздывания генератора использовался ключ $S$, управляемый внешним гармоническим сигналом $x(t)=A \sin (2 \pi f t)$ генератора (GS), где $A$ и $f-$ амплитуда и частота внешнего воздействия соответственно. Для оцифровки сигнала $x(t)$ использован аналогоцифровой преобразователь ADC-2. 


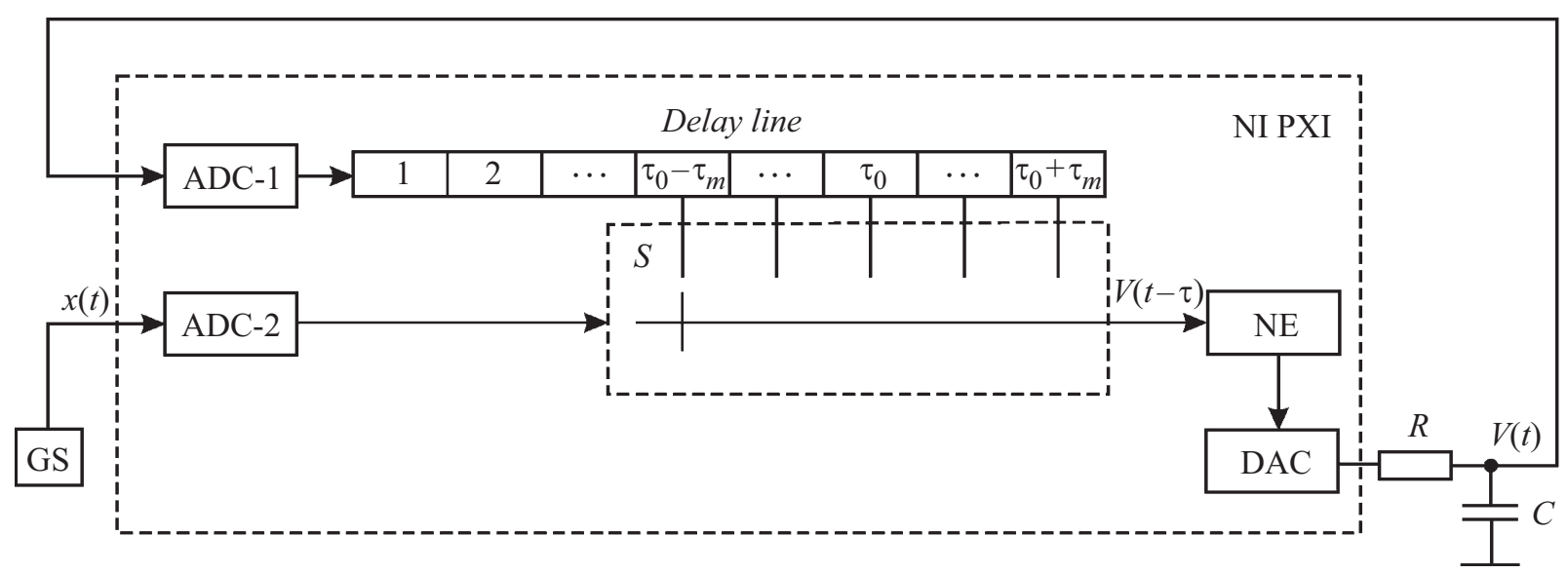

Рис. 1. Блок-схема экспериментальной установки. NE - нелинейный элемент, NI PXI - система ввода-вывода многоканальных данных, ADC-1 и ADC-2 - аналого-цифровые преобразователи, DAC - цифро-аналоговый преобразователь, $S$ - переключатель, GS - генератор гармонического сигнала.
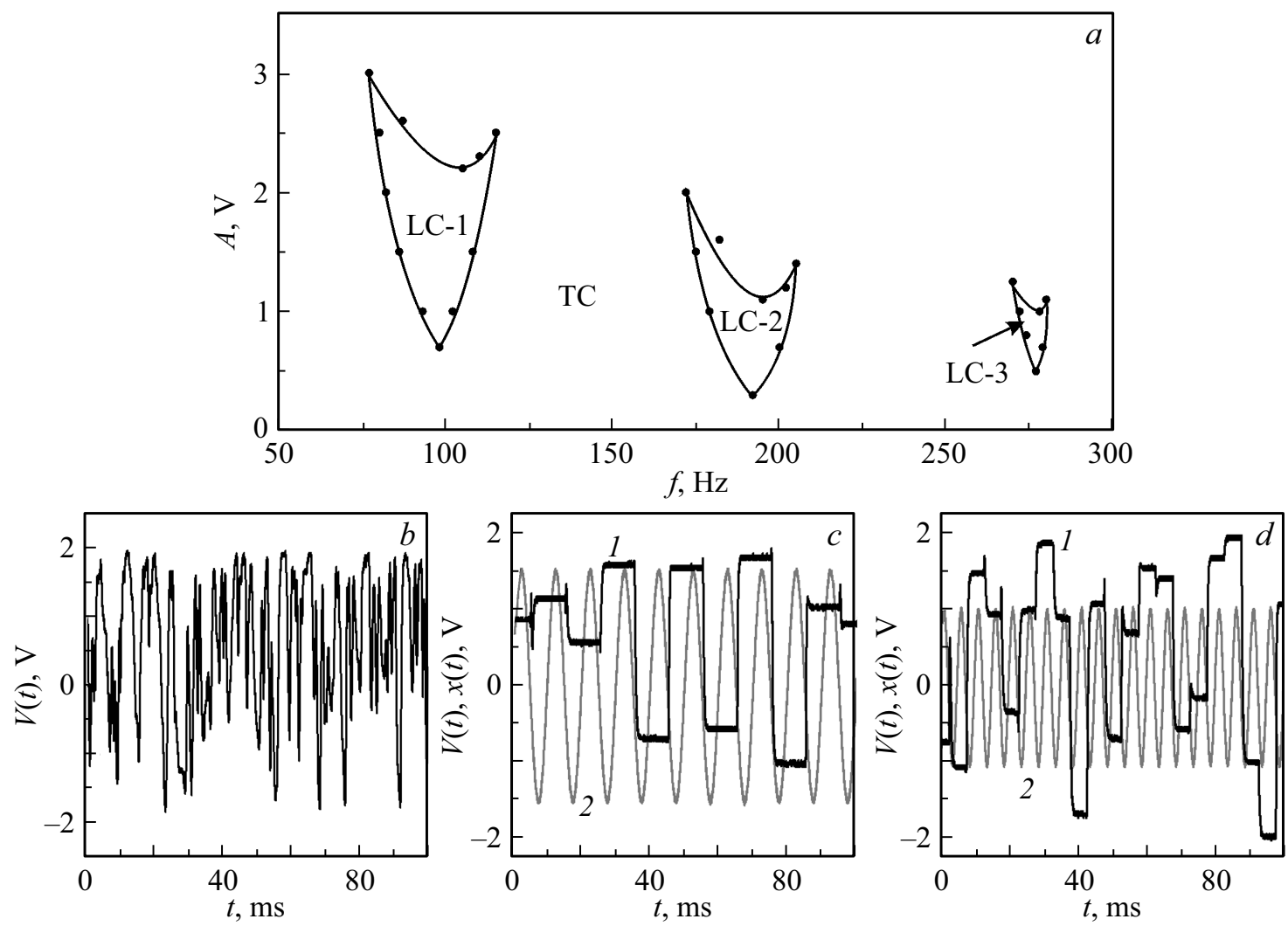

Рис. 2. $a-$ области существования турбулентного хаоса (TC) и ламинарного хаоса (LC) на экспериментальной плоскости параметров $(f, A)$ генератора $(1) ; b$ - характерная временна́я реализация колебаний $V(t)$ при турбулентном хаосе; $c$ - временны́е реализации сигналов $V(t)$ (линия 1$)$ и $x(t)$ (линия 2) в области ламинарного хаоса LC-1; $d$ - временны́е реализации сигналов $V(t)$ (линия 1) и $x(t)$ (линия 2) в области ламинарного хаоса LC-2.

Динамика генератора с переменным временем запаздывания описывается уравнением следующего вида:

$$
R C \dot{V}(t)=-V(t)+g(V(t-\tau(t))),
$$

где $V(t)$ и $V(t-\tau(t))$ - напряжения на входе и выходе линии задержки соответственно, $R$ и $C$ - сопротивле- ние и емкость элементов фильтра, $g$ - передаточная характеристика нелинейного элемента, $\tau(t)-$ время запаздывания, изменяющееся во времени по закону

$$
\tau(t)=\tau_{0}+k A \sin (2 \pi f t),
$$

$k$ - коэффициент, имеющий размерность $\mathrm{s} / \mathrm{V}, \mathrm{a} k A=\tau_{m}$. 


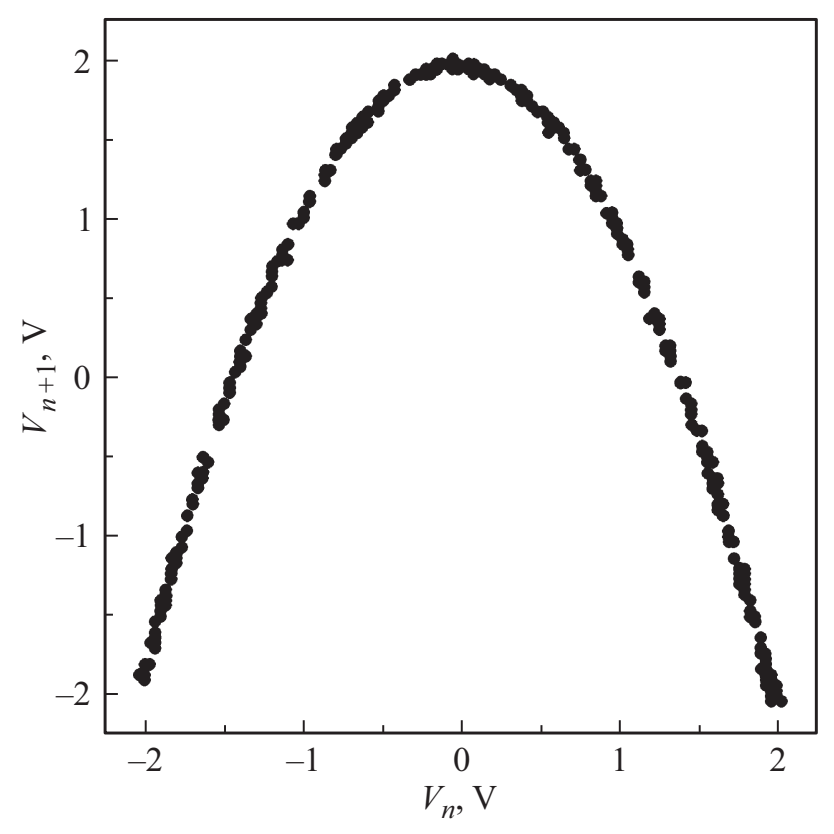

Рис. 3. Восстановленная нелинейная функция $g$ генератора (1) в области ламинарного хаоса LC-1.

Мы исследовали динамику генератора при $R C=0.2 \mathrm{~ms}$, $g(V)=\lambda-V^{2}$ (где параметр нелинейности $\lambda=1.99$ ), $\tau_{0}=10 \mathrm{~ms}, k=1 \mathrm{~ms} / 1 \mathrm{~V}$ и параметрах внешнего гармонического воздействия $A$ и $f$, изменяющихся от 0 до $3 \mathrm{~V}$ и от 50 до $300 \mathrm{~Hz}$ соответственно. Сигналы $V(t)$ и $x(t)$ оцифровывались аналого-цифровыми преобразователями с частотой $10 \mathrm{kHz}$, при этом время задержки $\tau_{0}$ соответствовало 100 отсчетам дискретного времени.

В отсутствие модуляции времени задержки $\tau_{0}$ внешним сигналом $(A=0)$ генератор с запаздывающей обратной связью демонстрирует при выбранных значениях параметров хаотические колебания $[12,18]$, соответствующие режиму турбулентного хаоса [15]. При периодически изменяющемся времени задержки (2) генератор (1) может демонстрировать качественно другой тип хаотического поведения, названный ламинарным хаосом $[15,16]$. На рис. 2, $а$ приведено разбиение экспериментальной плоскости параметров $(f, A)$ на области существования турбулентного хаоса (ТC) и различных режимов ламинарного хаоса (LC). В большинстве точек на плоскости $(f, A)$ генератор находится в режиме турбулентного хаоса. Характерная временна́я реализация колебаний $V(t)$ при турбулентном хаосе приведена на рис. $2, b$.

B замкнутых областях, обозначенных LC-1, LC-2 и LC-3 на рис. 2, $a$, в генераторе наблюдается ламинарный хаос, при котором ламинарные фазы периодически прерываются всплесками. В течение каждой ламинарной фазы величина $V(t)$ остается практически постоянной, а при переходе из одной ламинарной фазы в другую $V(t)$ меняется хаотически. Характерные временны́е реализации колебаний $V(t)$ в областях LC-1 и LC-2 представлены линиями 1 на рис. $2, c$ и $d$ соответственно. Линиями 2 показаны гармонические сигналы $x(t)$. Режимы ламинарного хаоса в областях LC-1, LC-2 и LC-3 различаются количеством ламинарных фаз $q$ на среднем времени запаздывания $\tau_{0}$. В области LC-1 на рис. 2, $a$ период внешнего воздействия $T=1 / f$ близок к величине $\tau_{0}$, при этом $q=1$ (рис. $\left.2, c\right)$. В области LC-2 период внешнего воздействия $T \approx \tau_{0} / 2$, при этом $q=2$ (рис. $2, d$ ). Область LC-3 на рис. 2, $a$, в которой $q=3$, имеет меньшую площадь, чем области LC-1 и LC-2, и ее сложнее наблюдать в эксперименте.

Обнаружено, что режим ламинарного хаоса возникает в генераторе (1) при периоде модулирующего сигнала $T \approx \tau_{0} / p$ (где $p$ - целое число) и относительно небольшой скорости изменения $\tau(t)$. Поскольку скорость изменения $\tau(t)$ растет с увеличением $f$ и с увеличением $A$, области ламинарного хаоса на плоскости $(f, A)$ замкнуты сверху и становятся более узкими с ростом $f$. При быстром изменении $\tau(t)$ режим ламинарного хаоса не наблюдается, по-видимому из-за переключений между мультистабильными состояниями генератора, в которых колебания совершаются либо на основной моде, либо на более высоких гармониках [4].

Обозначим постоянное значение $V(t)$ в течение $n$-й ламинарной фазы через $V_{n}$, а постоянное значение $V(t)$ в течение $(n+1)$-й ламинарной фазы - через $V_{n+1}$. Для режима ламинарного хаоса в области LC-1 на рис. 2, $a$ должно выполняться соотношение $V_{n+1}=g\left(V_{n}\right)$, в области LC-2 $V_{n+2}=g\left(V_{n}\right)$, а в области LC-3 $V_{n+3}=g\left(V_{n}\right)$ [16]. Поэтому в режиме ламинарного хаоса можно реконструировать нелинейную функцию $g$, построив соответствующую зависимость. На рис. 3 приведена зависимость $V_{n+1}$ от $V_{n}$ для области LC-1, достаточно хорошо совпадающая с истинной передаточной характеристикой $g$ нелинейного элемента генератора. В режиме турбулентного хаоса с переменным временем запаздывания реконструировать нелинейную функцию не удается.

Итак, нами впервые экспериментально исследовано явление ламинарного хаоса в генераторе с запаздывающей обратной связью с переменным временем задержки. Построено разбиение экспериментальной плоскости параметров частота-амплитуда внешнего гармонического воздействия, модулирующего время запаздывания генератора, на области существования различных режимов ламинарного хаоса. Показана возможность реконструкции нелинейной функции генератора с запаздывающей обратной связью в режиме ламинарного хаоса.

\section{Финансирование работы}

Работа выполнена при поддержке Российского фонда фундаментальных исследований (проект № 19-02-00071). 


\section{Конфликт интересов}

Авторы заявляют, что у них нет конфликта интересов.

\section{Список литературы}

[1] Kuang Y. Delay differential equations with applications in population dynamics. Boston: Academic Press, 1993. 398 p.

[2] Erneux T. Applied delay differential equations. N.Y: SpringerVerlag, 2009. $204 \mathrm{p}$.

[3] Lang R., Kobayashi K. // IEEE J. Quantum Electron. 1980. V. 16. P. 347-355. DOI: 10.1109/JQE.1980.1070479

[4] Ikeda K., Matsumoto K. // Physica D. 1987. V. 29. P. 223-235. DOI: $10.1016 / 0167-2789(87) 90058-3$

[5] Yanchuk S., Perlikowski P. // Phys. Rev. E. 2009. V. 79. P. 046221. DOI: 10.1103/PhysRevE.79.046221

[6] Пономаренко В.И., Кульминский Д.Д., Прохоров М.Д. // Письма в ЖТФ. 2018. Т. 44. В. 17. С. 11-18. DOI: 10.21883/PJTF.2018.17.46565.17366

[7] Senthilkumar D.V., Lakshmanan M. // Phys. Rev. E. 2007. V. 76. P. 066210. DOI: 10.1103/PhysRevE.76.066210

[8] Hart J.D., Bansal K., Murphy T.E., Roy R. // Chaos. 2016. V. 26. P. 094801 . DOI: $10.1063 / 1.4953662$

[9] Ponomarenko V.I., Kulminskiy D.D., Prokhorov M.D. // Phys. Rev. E. 2017. V. 96. P. 022209. DOI: 10.1103/PhysRevE.96.022209

[10] Faggian M., Ginelli F., Marino F., Giacomelli G. // Phys. Rev. Lett. 2018. V. 120. P. 173901. DOI: 10.1103/PhysRevLett.120.173901

[11] Rombouts J., Gelens L., Erneux Th. // Phil. Trans. Roy. Soc. A. 2019. V. 377. P. 20180127. DOI: 10.1098/rsta.2018.0127

[12] Пономаренко В.И., Прохоров М.Д. // Письма в ЖТФ. 2002. T. 28. B. 16. C. 37-44.

[13] Senthilkumar D.V., Lakshmanan M. // Chaos. 2007. V. 17. P. 013112. DOI: $10.1063 / 1.2437651$

[14] Martinez-Llinas J., Porte X., Soriano M.C., Colet P., Fischer I. // Nature Commun. 2015. V. 6. P. 7425. DOI: $10.1038 /$ ncomms 8425

[15] Müller D., Otto A., Radons G. // Phys. Rev. Lett. 2018. V. 120. P. 084102. DOI: 10.1103/PhysRevLett.120.084102

[16] Hart J.D., Roy R., Müller-Bender D., Otto A., Radons G. // Phys. Rev. Lett. 2019. V. 123. P. 154101. DOI: 10.1103/PhysRevLett.123.154101

[17] Кульминский Д.Д., Пономаренко В.И., Сысоев И.В., Прохоров М.Д. // Письма в ЖТФ. 2020. Т. 46. В. 4. С. 26-29. DOI: 10.21883/PJTF.2020.04.49046.18018

[18] Sysoev I.V., Ponomarenko V.I., Kulminskiy D.D., Prokhorov M.D. // Phys. Rev. E. 2016. V. 94. P. 052207.

DOI: 10.1103/PhysRevE.94.052207 